\title{
Added value of fetal MRI as a complementary method to antenatal ultrasound in the assessment of non-CNS fetal congenital anomalies
}

\author{
Samar Mohamed Eshiba ${ }^{1 *} \mathbb{D}$, Mohamed Hamdy Zahran ${ }^{1}$, Abdelaziz Mohamed Elnekeidy ${ }^{1}$,
} Tamer M. Abdeldayem ${ }^{2}$ and Hebatallah Hassan Mamdouh Hassan ${ }^{1}$

\begin{abstract}
Background: Birth defects and congenital anomalies are different words used to describe developmental abnormalities that occur at birth. Congenital anomalies diagnosis during pregnancy is a difficult topic to which ultrasonography has made significant contributions. The availability of a generally safe, independent technique in the evaluation of prenatal anomalies would be a welcomed clinical and scientific alternative. Ultrasound (US) is the predominant modality for evaluating disorders related to fetus and pregnancy. In most situations, this examination by a professional operator offers sufficient information about fetal morphology, surroundings, and well-being. The abnormalities revealed by ultrasound can be subtle or inconclusive at times. MRI has been demonstrated to be useful in such circumstances in various studies. So the effective use of fetal MRI in the evaluation of non-CNS abnormalities of the body is a reason for adopting fetal MRI as an adjunct to US in obstetric imaging. This study aimed to examine the role of fetal MRI as a complementary method to the antenatal US in assessing non-CNS anomalies and how it changed or modified the diagnosis of anomalies.
\end{abstract}

Results: By analyzing the data of 30 pregnant females with fetal non-CNS congenital anomalies, the diagnostic accuracy of prenatal ultrasound alone in the detection of congenital anomalies was $76 \%$, with a sensitivity of about $76 \%$. And diagnostic accuracy of MRI alone was $96.6 \%$, with a sensitivity of approximately $96.6 \%$. Moreover, the diagnostic accuracy of combined prenatal US and prenatal MRI in the detection of congenital anomalies was $100 \%$, with sensitivity about $100 \%$ and PPV about $100 \%$.

Conclusion: Fetal MRI raises confidence in non-CNS malformation assessment. Compared to US, MRI overcomes many of the obstacles faced by the antenatal US. MRI is superior to the US in refining, changing, or adding more diagnostic information about the disease.

Keywords: Magnetic resonance imaging, Ultrasound, Central nervous system

*Correspondence: s_eshiba@yahoo.com

1 Department of Diagnostic and Interventional Radiology, Faculty of Medicine, Alexandria University Hospital, Champollion Street, El Azareeta, Egypt

Full list of author information is available at the end of the article

\section{Background}

Ultrasound (US) is the first method in evaluating the fetus. In most situations, this examination by a professional operator offers sufficient information about fetal morphology and well-being. However, factors such as maternal obesity, suboptimal fetal position, multiple gestations, oligohydramnios, and the near-field 
reverberation artifact have a negative impact on US quality [1].

The abnormalities revealed by the US can be subtle or inconclusive at times. Magnetic resonance imaging (MRI) has been demonstrated to be useful in such circumstances in various studies [2].

Over the past decade, fetal MRI has become a powerful tool in fetal assessment, owing to superior tissue contrast, wide field of view, and relative independence on the operator. In the literature, most reports on fetal MRI are related to the central nervous system (CNS).

So the effective use of fetal MRI in the evaluation of non-CNS abnormalities of the body is a reason for adopting fetal MRI as an adjunct to the US in obstetric imaging [2].

After the implication of fetal MRI, in-utero fetal surgical intervention can accurately recognize the nature of the disease in certain anomalies, resulting in improvement of survival rates and functional consequences as in case of disorders related to twins, myelomeningocele, congenital diaphragmatic hernia, and urinary obstruction [3].

This study aimed to examine the role of fetal MRI as a complementary method to the antenatal US in assessing non-CNS anomalies and how it changed or modified the diagnosis of anomalies.

\section{Methods and technique}

This prospective study was conducted on 30 pregnant females out of ten thousand pregnant females for routine anomaly scan referred to maternity hospital of Alexandria provisionally diagnosed to have non-CNS congenital anomalies based on antenatal US examination and later on were referred to the MRI unit of the Radio-diagnosis Department at our institute for further diagnostic confirmation in a time interval from August 2018 to December 2020. The local scientific review board committee approved this study. From all participating ladies, informed written consent was obtained.

The following were excluded from this study: (a) first trimester pregnancy, (b) cardiac and pure CNS anomalies, (c) patient in labor, and (d) contraindications for MRI study: metallic implants, anxiety disorders, and severe claustrophobia.

After thorough history taking about the patient's previous history of abortion or previous congenital anomalies, age, and risk factors for congenital fetal anomalies, the radiological assessment was performed.

\section{Imaging procedures}

Expert diagnostic antenatal US and fetal MRI were done for all patients.

\section{The antenatal US}

The US imaging was performed by two experienced sonographers specialized in the prenatal US: One was an obstetrician (about 15 years experience) and the other was a radiologist (about 10 years experience). Both were blinded from each other results; however, they reached the same diagnosis in consensus in each case which was again confirmed by two other experienced radiologist and obstetrician to perform the final registration data.

\section{MRI}

Once the US suspected any congenital non-CNS anomaly, MRI was routinely performed at the second or third trimester between (18-36) weeks of gestation, as before the age of 18 weeks fetal MRI does not usually provide any further information than an ultrasound scan; however [4], most organs can be seen in detail between 26 and 32 weeks of pregnancy, when disorders associated with abnormal development have developed, although each pregnancy and fetus is unique.

Fetal MRI was done using a 1.5-T whole-body system (Ingenia, Philips, Healthcare, Best, the Netherlands), using a 12-channel pelvic-phased arrayed coil.

\section{Imaging protocol}

The position of the mother was supine or left lateral. Neither sedation nor intravenous contrast agent was given. An ultrafast T2-weighted sequence was used to obtain the initial localizer in orthogonal planes to the maternal axis to localize the fetus and identify the pathology. For optimum image quality, the region of interest (ROI) was selected in the coil center. Following fetal imaging planes, further image acquisition was made. The sequences routinely applied to all participants using standard parameters are detailed in Table 1.

The affection of the image quality by fetal movement necessitates a repetition of the sequence. Two experienced radiologists with 10 and 5 years of experience used an advanced MRI workstation (IntelliSpace Portal) to process and interpret the image stacks blinded to the prenatal US diagnosis.

Each radiologist processed and interpreted the images independently. The inter-observer agreement was closely perfect agreement (kappa $=0.87$; 95\% CI $0.60-0.99$ ). After independent reviews, a further round of discussion was held, and agreement was obtained for resolving any minor discrepancy concerning the final interpretation.

Non-lethal anomalies in survivors were diagnosed using a postnatal radiological study (US/MRI/CT); nevertheless, in cases of fatal malformations, as an 
Table 1 Parameters in fetal MRI sequence

\begin{tabular}{|c|c|c|c|c|c|c|}
\hline Sequence & $\mathrm{TR}(\mathrm{ms})$ & TE (ms) & $\begin{array}{l}\text { Slice thickness/ } \\
\text { gab }(\mathrm{mm})\end{array}$ & matrix & FA (degree) & Plane \\
\hline Single-shot fast SE sequence (SSFSE) & 10,000 & 100 & $3-4 / 0.0 .5$ & $169 / 256$ & 90 & $\begin{array}{l}\text { Axial } \\
\text { Sagittal } \\
\text { Coronal }\end{array}$ \\
\hline T1-weighted (T1-WI) & $100-140$ & 4 & $4-6 / 0.2-0.4$ & $160 / 256$ & 70 & Changeable \\
\hline $\begin{array}{l}\text { Balanced steady-state free precession } \\
(\mathrm{SSFP})=\text { balanced fast field echo (b-FFE) }\end{array}$ & $3.5-4$ & $1.7-2$ & $4 / 0$ & $256 / 256$ & 80 & Changeable \\
\hline Diffusion-weighted image (DWI) & 1470 & 125 & $5 / 0.1$ & $108 / 256$ & 90 & Axial \\
\hline
\end{tabular}

autopsy was not possible owing to religious restrictions, MRI diagnosis was regarded as the gold standard for diagnosis.

Data collection, coding, and revision were done, followed by data entrance to the Statistical Package for Social Science (Rstudio) version 2.3.2. The number and percentage presented the qualitative data. In comparison, the quantitative data with parametric distribution were presented by mean, standard deviations (SD), and ranges, and the quantitative data with nonparametric distribution were presented by a median with interquartile range (IQR) for the quantitative data with the nonparametric distribution.

\section{Results}

A total of 30 pregnant females with multiple non-CNS anomalies out of ten thousand pregnant females were investigated and enrolled in our research work with a mean age of 27 years \pm 5 years. The youngest participant was 18 years, and the eldest was 40 years of age. The gestation ages of examined fetuses ranged from 18 to 36 weeks.

Considering the different systems affected, the gastrointestinal anomalies was the most common in our study (11/30) $36 \%$ cases followed by renal anomalies (8/30) 26\% cases, then thoracic anomalies (5/30) $16.5 \%$ cases then neck and facial anomalies (4/30) $13.3 \%$ cases and (2/30) $6.5 \%$ cases of twin pregnancy with multiple congenital anomalies as in case of asymmetric conjoint twin with fusion anomalies and GIT anomalies.

\section{Regarding the GIT anomalies}

Three cases with duodenal atresia were detected on ultrasound, and MRI confirmed the diagnosis. A case of intrahepatic tumor was detected by ultrasound as suspected hepatic tumor and MRI confirmed the diagnosis and the extension of the tumor. In addition to a case with splenic cyst misdiagnosed by ultrasound as adrenal cyst, but MRI changed the diagnosis to splenic cyst (Fig. 1). On the other hand, a case with hepatic Caroli disease was diagnosed by MRI in addition to CNS anomalies which was the main indication in performing the MRI, and after reviewing the ultrasound, it was not visualized. A case with esophageal atresia was suspected on ultrasound due to absent gastric bubbles, and MRI confirmed the diagnosis. Four cases with omphalocele were identified by ultrasound, and MRI confirmed the diagnosis moreover, MRI was superior in assessment of the extent of herniation of the solid organs and the intestines out of the abdominal cavity.

\section{Concerning cases with genitourinary anomalies}

A case with adrenal neoplastic process was detected and misdiagnosed on ultrasound as renal tumor (Fig. 2) but MRI changed the diagnosis to the adrenal neoplastic lesion. Moreover, two cases of infantile polycystic kidneys (Fig. 3) were detected: one of them was misdiagnosed on ultrasound as non-visualized kidneys due to marked oligohydramnios and dysmorphic fetus and MRI changed the diagnosis to infantile polycystic kidneys. Also, a case with a posterior urethral valve (PUV) was diagnosed by ultrasound and confirmed by MRI as a posterior urethral valve with bilateral hydro-nephrosis. Three cases

(See figure on next page.)

Fig. 133 -year-old pregnant female at 28 weeks of gestation underwent ultrasound followed by fetal MRI to exclude congenital anomalies. Prenatal ultrasound: $\mathbf{a}, \mathbf{b}$ Ultrasound revealed small cystic lesion at the retro-gastric region and superior to the left kidney... suspected to be adrenal in origin (arrow). Prenatal fetal MRI sequences revealed retro-gastric small cyst of fluid signal intensity at the anatomical site of spleen (white arrow) effacing the left suprarenal gland and left kidney (black arrow) which are seen separable with preserved zonal anatomy: c axial T2-weighted SSFSE, d Sagittal T2-weighted SSFSE, e coronal T2-weighted SSFSE, f axial T1-weighted ultrafast gradient echo. Postnatal ultrasound: $\mathbf{g}$ ultrasound confirmed small congenital splenic cyst 


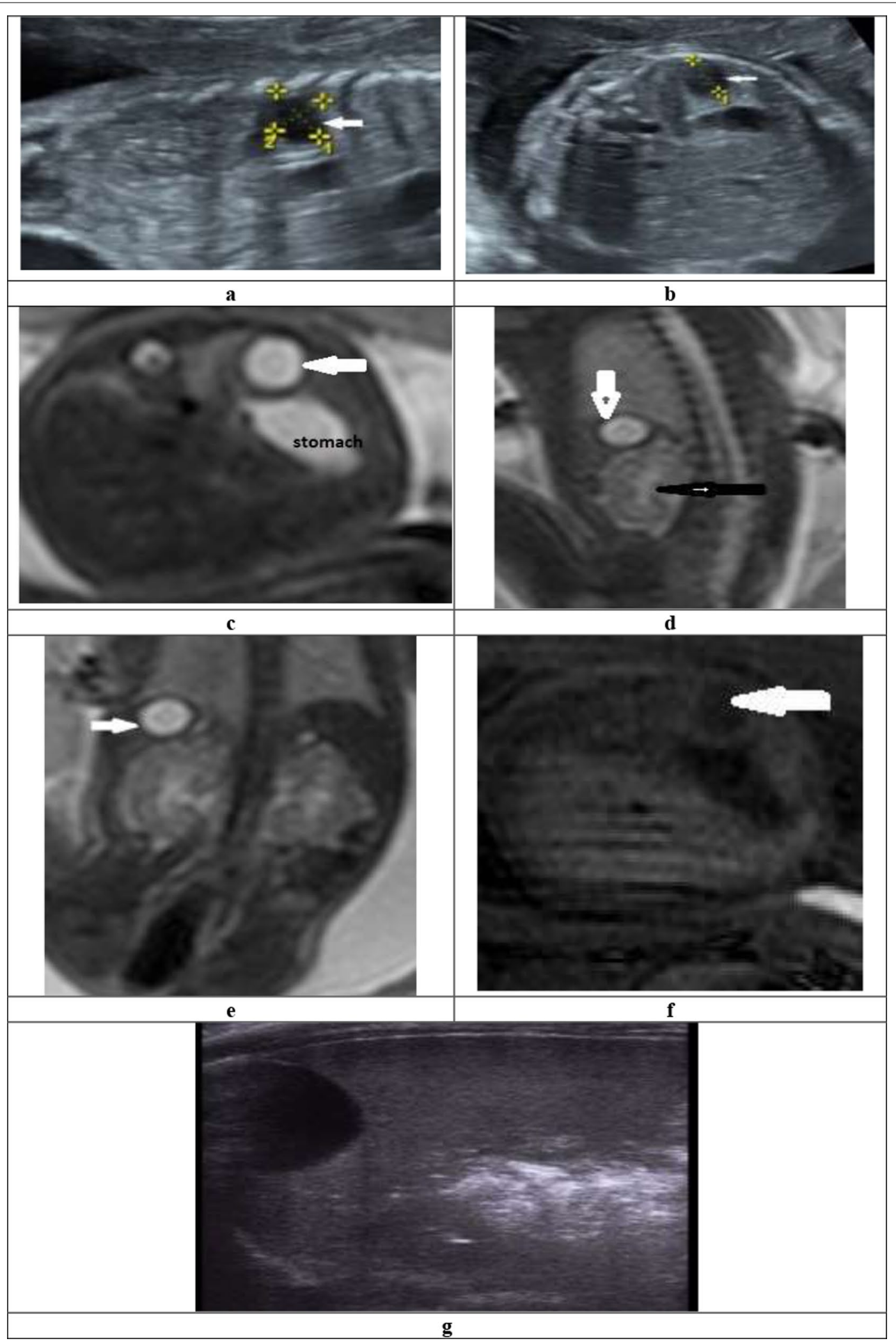

Fig. 1 (See legend on previous page.) 
with renal agenesis were suspected by ultrasound, and MRI proved the diagnosis. On the other hand, a case of the multicystic dysplastic kidney was diagnosed correctly by ultrasound but misdiagnosed by MRI as pelvi-ureteric junction obstruction (PUJO).

\section{Regarding thoracic anomalies}

Four cases of congenital diaphragmatic hernia (CDH) were identified, one of them misdiagnosed on ultrasound as esophageal atresia, and MRI changed the diagnosis to congenital diaphragmatic hernia (Fig. 4). The other three cases were identified by ultrasound and established by MR. Also, a case of congenital lobar emphysema was misdiagnosed on ultrasound as congenital cystic adenomatoid malformation (CCAM) and MRI changed the diagnosis to congenital lobar emphysema (Fig. 5).

\section{Facial anomalies according to prenatal MRI}

There was only one case of cleft lip and palate $(\mathrm{CL} / \mathrm{P})$ identified by ultrasound and confirmed by MRI as a better assessment of the palatal defect and excluded other anomalies.

\section{Concerning cases with neck anomalies}

There were three cases with congenital neck anomalies. One of them was cervical teratoma (Fig. 6) which was discovered by ultrasound and confirmed by MRI, while the other two cases were cystic hygroma correctly detected by the US and proved by MRI.

Concerning the twin pregnancy, there were two cases with twin pregnancy; one of them was twin pregnancy with duplicated heads associated with CNS anomalies and non-CNS anomalies identified by US and MRI confirmed the diagnosis, while the other case of asymmetrical conjoint twin pregnancy was diagnosed by ultrasound and MRI established the diagnosis with a better assessment of fusion anomalies than ultrasound.

Of the 30 pregnancies, correlation of MRI diagnosis of fetal malformations with prenatal US findings resulted in three groups (Table 2):
1. 'Agreed' in five fetuses (16.6\%): Fetal MRI correctly confirmed prenatal US diagnosis without additional findings.

2. 'Modified' in 24 fetuses (80\%): Fetal MRI correctly added additional findings in 19 cases and modified or changed prenatal ultrasound diagnosis in five cases

3. 'Missed' in one fetus (3.5\%): Fetal MRI missed correct findings identified by the prenatal US, as in the case of unilateral multicystic dysplastic kidney.

According to this study, the diagnostic accuracy of prenatal ultrasound alone in the detection of congenital anomalies was $76 \%$, with a sensitivity of about $76 \%$. And diagnostic accuracy of MRI alone was $96.6 \%$, with a sensitivity of approximately $96.6 \%$. Moreover, the diagnostic accuracy of combined prenatal US and prenatal MRI in the identification of congenital abnormalities was $100 \%$, with sensitivity about $100 \%$ and PPV about $100 \%$ (Table 3).

\section{Discussion}

Nowadays, fetal anomaly scanning relies more on MRI technology. It has gone from being a supplemental modality to being an essential part of fetal imaging procedures in the past several years [5].

Fetal MRI is usually used if the fetal US revealed a suspicious finding or if the sonographically detected fetal abnormalities necessitate further evaluation.

We compared the diagnostic accuracy of prenatal MRI to that of the US in identifying non-CNS anomalies between 18 and 36 weeks of gestation.

According to our study, the sensitivity of prenatal US alone in the detection of congenital anomalies was inferior to the sensitivity of MRI alone, and this is in accordance with Gonçalves et al. [6] study that reported higher sensitivity of MRI more than that of 2DUS and 3DUS.

As regards diagnostic accuracy, several studies revealed superiority of MRI in identifying congenital fetal anomalies compared to the US [7]. This was in agreement with our study that showed higher accuracy for MRI than for the US.

In our study, in $5 / 30$ cases (16.6\%), the US made the diagnoses and proved them by MRI. There were 24/30 cases $(80 \%)$ in which MRI imaging provided more

(See figure on next page.)

Fig. 2 25-year-old pregnant female at 35 weeks of gestation, with suspected renal anomalies. Prenatal ultrasound: a, b revealed sizable intra-abdominal mass indenting the liver occupying the right renal fossa...? renal in origin. Prenatal fetal MRI sequences revealed sizable soft tissue iso to hyperintense lesion at the anatomical site of right adrenal fossa (solid arrow) seen indenting the posteroinferior surface of the liver anteriorly and displacing still separable from the right kidney (hollow arrow) with no hemorrhagic foci as in T1 excluding hemorrhage .... features of sizable right suprarenal lesion (? neuroblastoma) ... c c d Axial T2 SSFSE sequence, e sagittal T2 SSFSE sequence, $\mathbf{f}$ axial DWI sequence, $\mathbf{g}$ axial T1 gradient echo sequence. Diagnosis was sizable right suprarenal lesion (? neuroblastoma) 


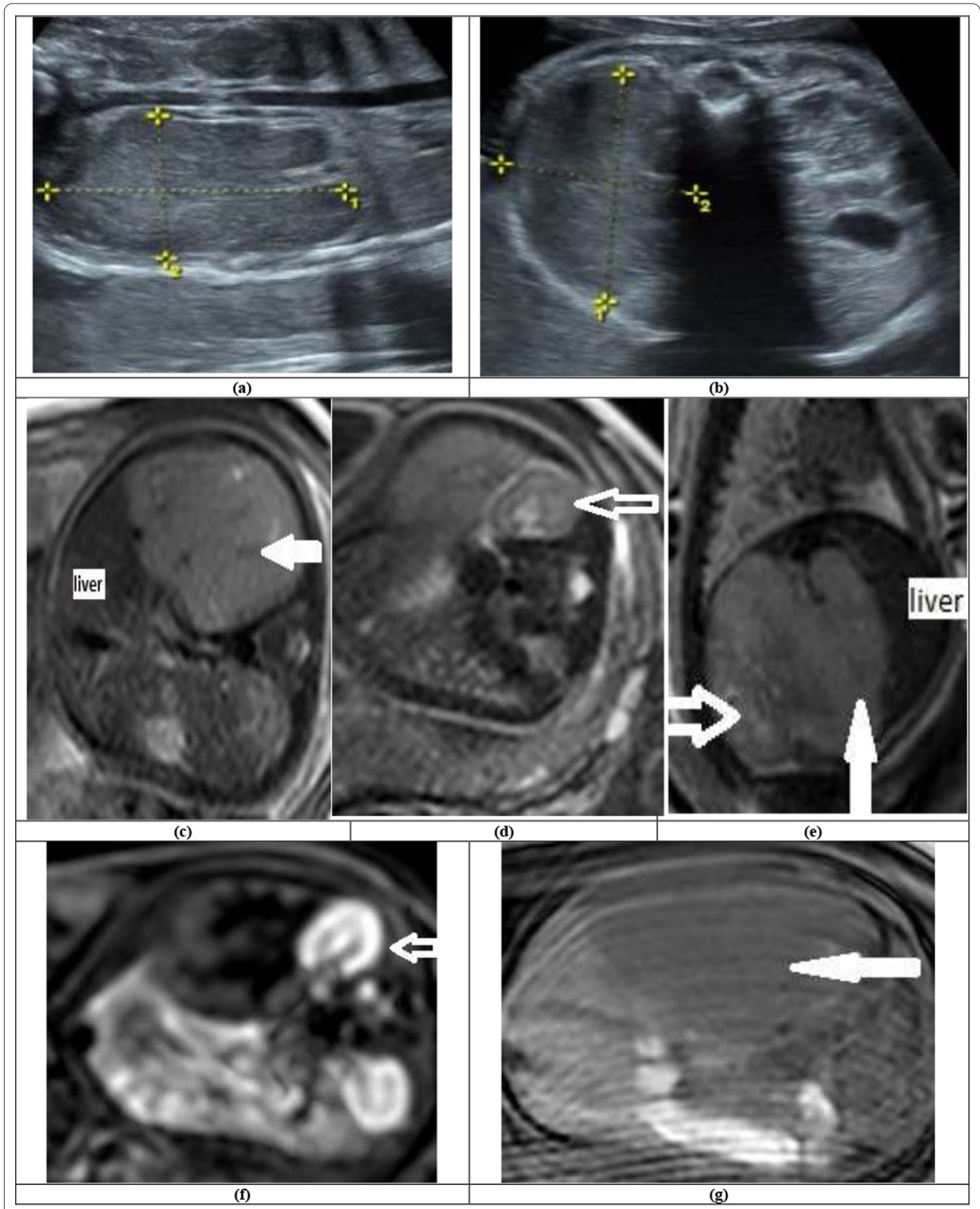

Fig. 2 (See legend on previous page.) 


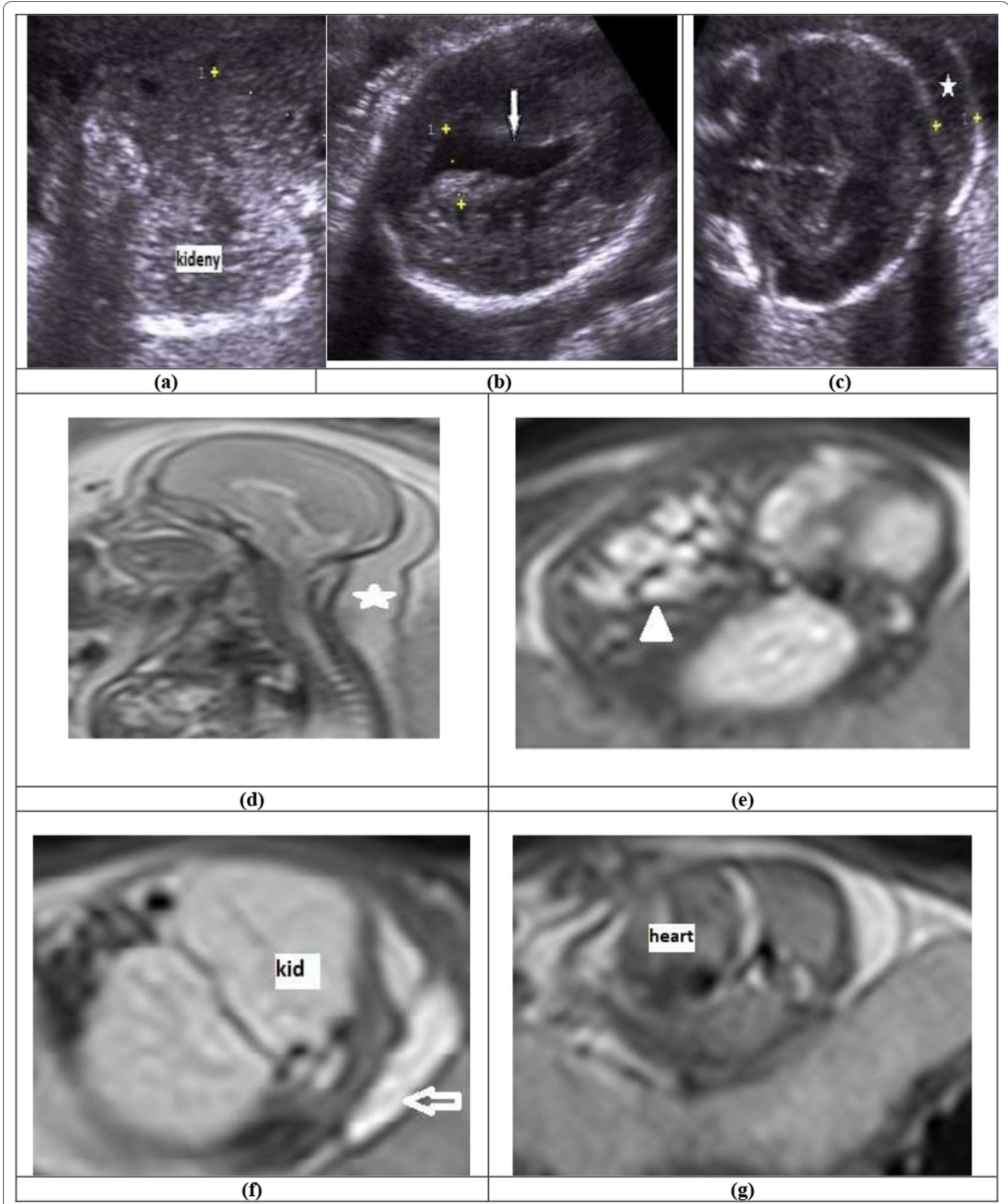

Fig. 3 25-year-old pregnant female at 21 weeks of gestation presenting with oligohydramnios. Prenatal ultrasound: a-c revealed bilateral enlarged echogenic kidneys associated with ectatic lateral ventricles (white arrow) and shallow posterior fossa with increased nuchal fold (asterisk). Prenatal fetal MRI sequences revealed bilateral enlarged kidneys (infantile polycystic kidneys). Associated with IHBD (Caroli disease) (arrow head), spina bifida with meningocele (hollow arrow), lung hypoplasia and diffuse increase in nuchal fold ... over all features matching with Meckel-Gruber syndrome: d sagittal $\mathbf{e}-\mathbf{g}$ axial T2-weighted SSFSE sequence. Diagnosis was Meckel-Gruber syndrome 


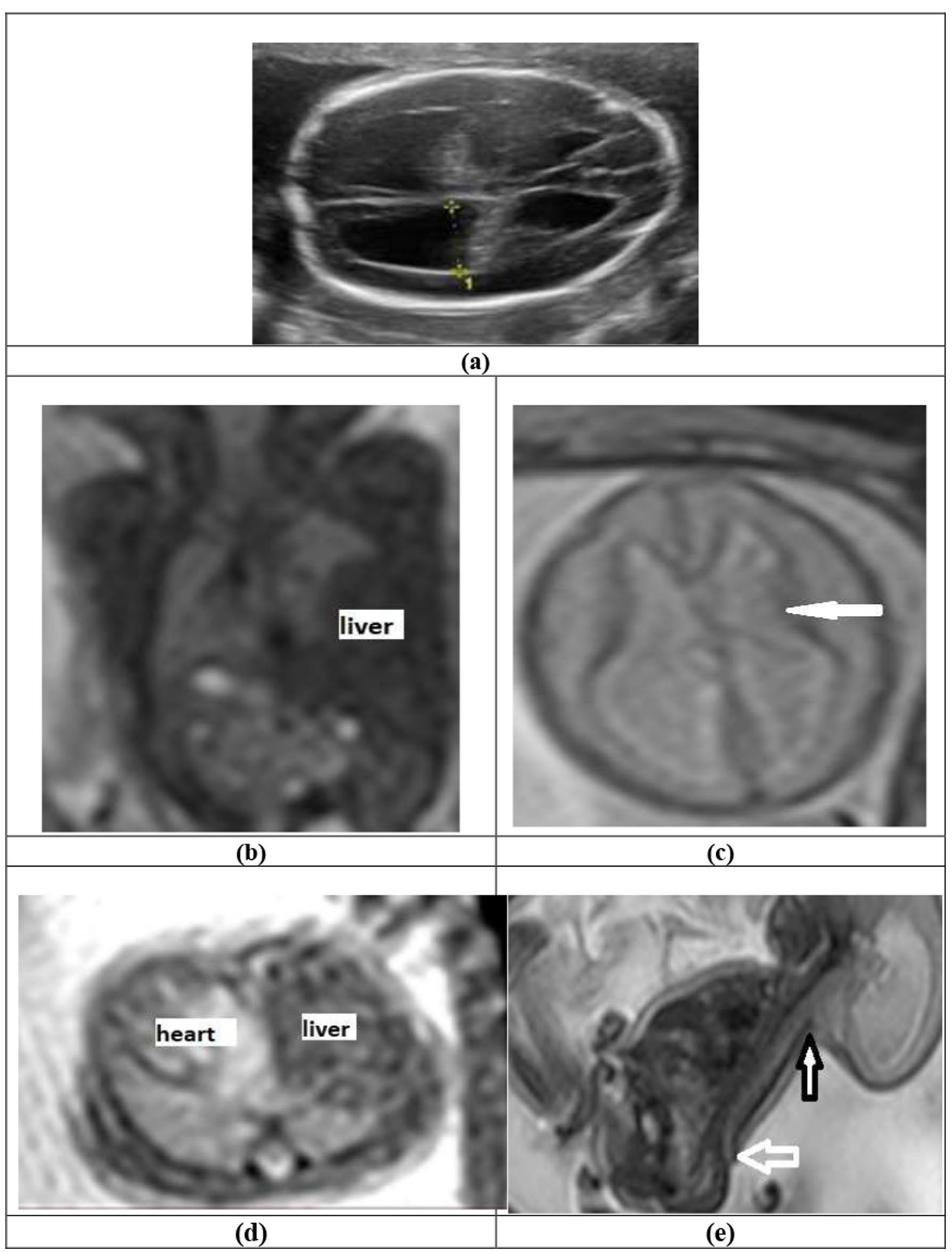

Fig. 4 28-year-old pregnant female at 25 weeks of gestation presented with polyhydramnios. Prenatal ultrasound: a revealed supra-tentorial ventricular dilation. Prenatal fetal MRI sequences revealed supra-tentorial ventricular dilation (white arrow) with shallow posterior fossa (black arrow) and spinal spina bifida (hollow arrow) at lumbosacral spine ... Chiari II malformation, and congenital diaphragmatic hernia with herniating part of liver in the right hemi-thorax, $\mathbf{b}$ coronal T2 SSFSE sequence of the trunk, $\mathbf{c}$ axial T2 SSFSE sequence of the brain, $\mathbf{d}$ axial balanced steady-state free precession sequence (b-FFE) of the chest, e sagittal T2 SSFSE sequence of the spine. Diagnosis was Chiari II malformation and congenital diaphragmatic hernia 


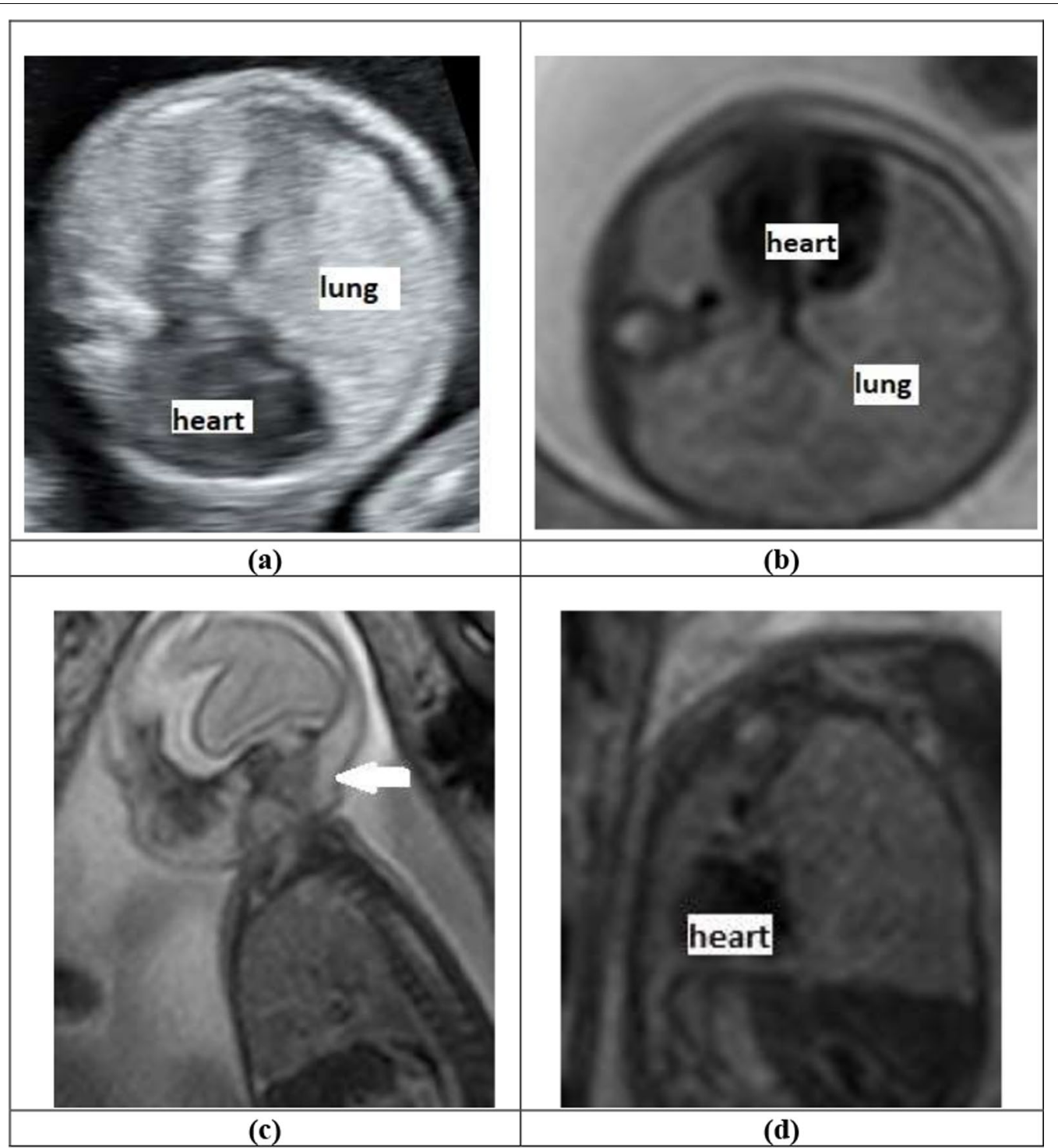

Fig. 5 25-year-old pregnant female at 27 weeks of gestation presenting with polyhydramnios. Prenatal ultrasound: a revealed polyhydramnios associated with enlarged echogenic lung ...? CCAM. Prenatal fetal MRI sequences revealed polyhydramnios and hyperinflated lung with stretched vessels with marked mediastinal shift (heart) to contralateral side and compressed contralateral lung with no evidence of cystic lesion associated with increase nuchal fold (white arrow) and flattening of the diaphragm ...features of congenital lung lobar emphysema (overinflation). b Axial T2 SSFSE sequence. $\mathbf{c}$ Sagittal T2 SSFSE sequence. d Coronal T2 SSFSE sequence. Diagnosis was congenital lung lobar emphysema

information than the US, which modified the diagnosis and, hence, the postnatal management plan. Still, MRI missed the correct diagnosis in $1 / 30$ cases $(3.5 \%)$ revealed by ultrasound as a case of unilateral renal cystic dysplastic kidney, even though MRI detected a renal pathology, still misdiagnosed it as PUJO, which was established postnatal as unilateral cystic dysplastic kidney.

Matching with Hosny IA et al. in 2010 [8] investigated 25 pregnant females with US followed by MRI after discovering congenital fetal anomalies. In $8 \%$ of cases
( 2 out of 25), the diagnosis was modified after using MRI because of the recognition of additional findings. MRI confirmed the diagnosis of US in the remaining $18 / 25(72 \%)$ cases.

\section{Gastrointestinal anomalies}

It is important for timing of fetal GI MR imaging studies, which should be scheduled at a gestational age when one would expect a normal distribution of meconium. Meconium should be seen in the left colon by 24 weeks gestation and is usually identified in the entire colon by 


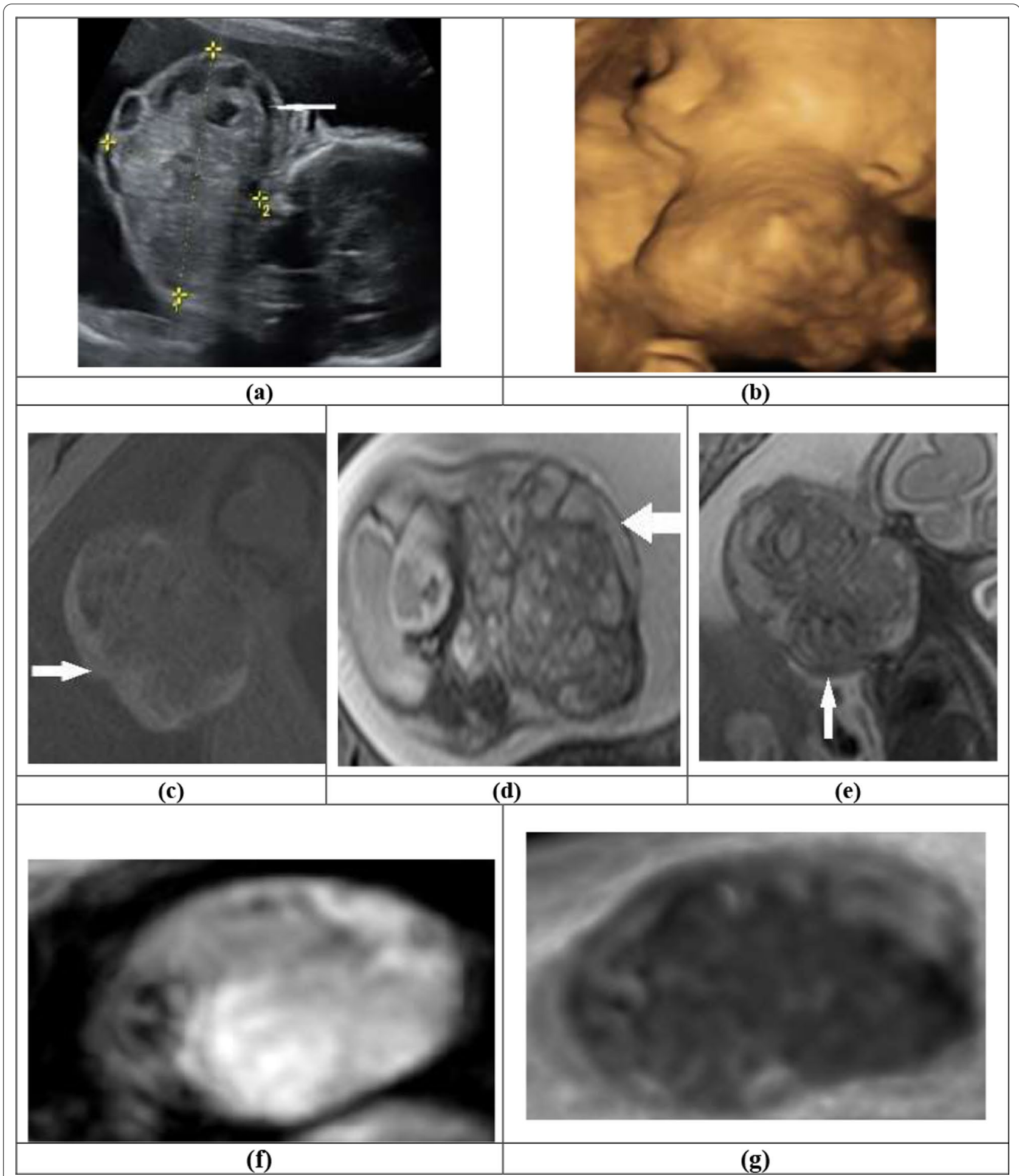

Fig. 6 20-year-old pregnant female at 26 weeks of gestation, prenatal ultrasound revealed polyhydramnios. Prenatal ultrasound: $\mathbf{a}$, b revealed complex solid and cystic neck swelling (arrow) with no detected vascularity involving the left side of the neck ...? ?cervical teratoma. Prenatal fetal MRI sequences revealed polyhydramnios and hyperextension of the neck and sizable neck swelling with solid and cystic changes (white arrow) with areas of hemorrhage (white arrow) seen compromising and obstructing the oropharyngeal airways with non-visualized stomach .... matching with congenital cervical teratoma: c sagittal T1-weighted image, $\mathbf{d}$ axial T2-weighted SSFSE sequence, e sagittal T2-weighted SSFSE sequence, f DWI, g ADC image. Diagnosis was congenital cervical teratoma 
Table 2 Results of prenatal MRI about prenatal US finding

\begin{tabular}{|c|c|c|c|}
\hline Results & Number & Percent (\%) & Cases \\
\hline Agreed & 5 & $16.6 \%$ & $\begin{array}{l}\text { Cleft lip } \\
\text { Duodenal atresia } \\
\text { Omphalocele }\end{array}$ \\
\hline Modified/changed & 24 & $80 \%$ & $\begin{array}{l}\text { Adrenal mass } \\
\text { Esophageal atresia } \\
\text { Caroli disease } \\
\text { Splenic cyst } \\
\text { Lobar emphysema } \\
\text { CDH } \\
\text { Cervical teratoma } \\
\text { Meckel gurber syndrome } \\
\text { Duodenal atresia } \\
\text { Omphalocele } \\
\text { Renal agenesis } \\
\text { Asymmetric conjoint twin } \\
\text { Cystic hygroma } \\
\text { PUV }\end{array}$ \\
\hline Missed & 1 & $3.5 \%$ & Cystic dysplastic kidney \\
\hline
\end{tabular}

25 weeks gestation, so better after 24 weeks of gestation [9].

Regarding cases with anterior abdominal wall defects (Gastroschisis and omphalocele) are two of the most prevalent types of anterior abdominal wall abnormalities. The umbilical cord insertion lies on the apex of the omphalocele, which is a midline supra-umbilical abdominal wall defect with herniation of abdominal content and peritoneum. Gastroschisis usually affects small intestinal loops, which are herniated and floating with no covering membrane. Gastroschisis may be accompanied by oligohydramnios and intrauterine growth restriction [10].

Our study showed four cases of omphalocele. MRI was better in the assessment of the extent of herniation of the solid organs and the intestines out of the abdominal cavity and useful to assess the abdominal wall defect more precisely. MRI easily identified the amniotic sac covering and the herniated contents, which are typical of omphalocele. It easily identified other associated anomalies such as CNS anomalies and assessed the lung in case of giant defect.

In cases of duodenal atresia, duodenal atresia is considered one of the most frequent causes of fetal bowel obstruction. The incidence of duodenal atresia reached up to one in 10,000 births in normal karyotype fetuses.

Because an association was found between duodenal atresia and Trisomy 21 in up to $33 \%$ of cases and also with the VACTERL, fetal MRI may be valuable to detect and characterize these associated anomalies (anorectal malformation, cardiac disease, renal anomalies, vertebral anomaly, trachea-esophageal fistula, limb anomalies) [11].

Our study showed three cases presenting with duodenal atresia, two cases diagnosed by ultrasound with no additional findings on MRI, and one case showed double bubble sign on ultrasound still MRI proved to be of added value as suggested duodenal stenosis rather than atresia as it was able to assess the distal bowel loops excluding distal bowel atresia or malrotation which was confirmed postnatal.

In the setting of esophageal atresia in our study, MRI confirmed the diagnosis of esophageal atresia with better evaluation of the esophageal pouch than ultrasound.

In situations with a small or non-visualized stomach, especially if associated with polyhydramnios, fetal US imaging can diagnose esophageal atresia. However, MRI provides a more accurate diagnosis of esophageal atresia, showing the contracted stomach easily. It is important to mention that other anomalies in the VACTERL association should be suspected in the case of esophageal atresia [9].

Regarding the hepatic and splenic pathology in our study, MRI was superior to ultrasound in the diagnosis of a splenic cyst, assessment of extension of the hepatic tumor, and intrahepatic dilation (Caroli disease).

This matched with Chen CP, Cheng SJ, Sheu JC et al. reported the added value of MRI in diagnosing the choledochal cyst prenatally [12]. MRI is more accurate than the US in diagnosing exophytic behavior of large hepatic and splenic cysts and delineating their relation with the surrounding parenchyma [12].

\section{Genitourinary anomalies}

The genitourinary structures can already be correctly identified and examined from about 20 weeks onwards with MRI [13]

Regarding cases of Renal agenesis, Our study matched with several previous which demonstrated the MRI's usefulness in assessing a wide range of fetal urinary anomalies.

In our study, in three fetuses, MRI confirmed the findings of US concerning non-visualization of kidneys and non-filling of the urinary bladder. Fetuses who are diagnosed with bilateral renal agenesis are incompatible with life. The parents' decision to terminate the pregnancy is supported by prenatal proof of bilateral renal agenesis. In such circumstances, severe oligohydramnios or anhydramnios are almost always present, and the US assessment is inaccurate. In comparison, MRI can be very useful in evaluating bilateral renal agenesis [14].

The absence of the bright signals in renal fossa can be detected easily by DWI sequence [14], which plays an important role in confirming the diagnosis. Bilateral renal agenesis is frequently associated with pulmonary hypoplasia, which also can be detected more accurately on MRI.

Regarding cases with Cystic kidneys in our study, cystic kidneys were shown in one fetus with unilateral 


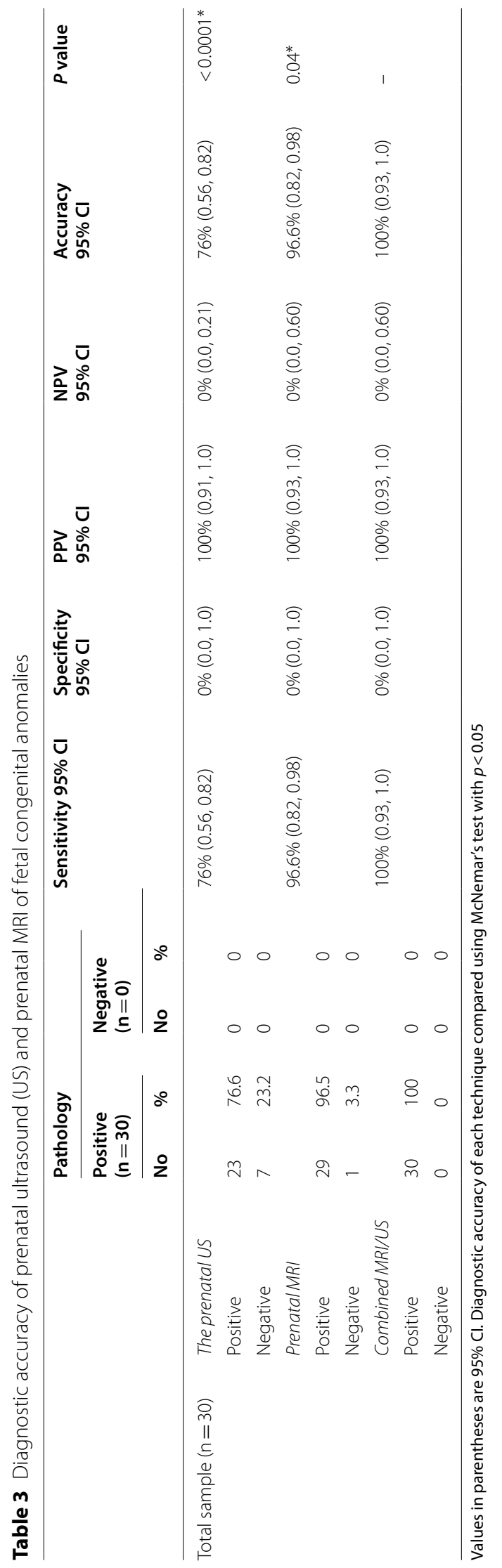


multicystic dysplastic kidneys and two fetuses with bilateral autosomal recessive polycystic kidneys (ARPKD), representing one of the most frequent urinary abnormalities. As the recurrence rate of ARPKD represents $25 \%$ while that MCDK represents $3 \%$, it is important to differentiate between both abnormalities [15]. When small cysts and marked enlargement of both kidneys are detected, the suspension of ARPKD is raised more than MCDK [15].

In two fetuses, MRI confirmed the US diagnosis of bilateral ARPKD. Fetal MRI showed obvious bilateral renal enlargement with a mottled hyperintense signal on T2-weighted images reflecting the innumerable small cysts in ARPKD [16]. When bilateral renal abnormalities and oligohydramnios are diagnosed, syndromal affection and other fetal anomalies are suspected such as MeckelGruber syndrome (MKS) $[17,18]$.

Concerning cases of pelvi-ureteric junction obstruction (PUJO), Urinary system obstruction at the pelvi-ureteric junction (PUJ), ureterovesical (U-V) junction, and/or urethra can be presented as a fetal abdominal cystic mass [19]. Correct diagnosis of urinary tract obstruction was made by fetal MRI in one fetus with accurate determination of the obstruction level. Due to extended view field, MRI delineated the massively dilated urinary bladder (megacystis) correctly in a fetus in our study; both dilated posterior urethra (Keyhole sign) and thickening of the wall of the urinary bladder contribute to the confirmation of the prenatal PUV diagnosis in this fetus [20].

\section{Thoracic anomalies}

Fetal MRI is a valuable tool in assessing chest malformation due to the high ability of T2WI MRI to visualize the anatomy and morphology of lung parenchyma. In addition, in the last few years, DWI can assess the physiological maturation of lung parenchyma throughout different gestational ages. It is proposed that the increase in the gestational age of pregnancy is accompanied by an increase in the ADC values of fetal lung parenchyma [21].

The normal fetal lung is homogenous and has a moderately high signal intensity of T2-weighted images. With maturation of the lungs, the signal intensity of the lungs increases, so better assessment of lung pathology after 24 weeks of gestation [21].

In the case of congenital diaphragmatic hernia, Out of four cases with a congenital diaphragmatic hernia $(\mathrm{CDH})$, three of them were identified by ultrasound, and MRI confirmed the diagnosis with more additional information regarding the content and associated lung hypoplasia. One case was misdiagnosed on ultrasound as esophageal atresia, and MRI changed the diagnosis.

The commonest site of $\mathrm{CDH}$ is the posterior aspect of the left hemidiaphragm. $\mathrm{CDH}$ results from a failure in diaphragmatic leaflet development. In fetuses with leftsided $\mathrm{CDH}$, the presence of the liver in the chest is evidence of a very poor prognosis. MRI can visualize liver position directly and distinguish meconium-filled herniated bowel loops from cystic lesions within the chest [22].

In the case of congenital lobar over inflation, prenatal fetal MRI has a great capability of diagnosing fetal thoracic malformations, such as congenital diaphragmatic hernia, extra lobar bronchopulmonary sequestration, congenital pulmonary airway malformation, congenital lobar emphysema, etc. Sometimes, the early exploration of the disease allows better patient counseling, pregnancy and delivery management, and neonatal care preparation [23]. As in our study, fetal MRI was superior to ultrasound in diagnosing congenital lobar over inflation.

\section{Neck and facial anomalies}

Although fetal MRI of the face, and neck can be performed during the second or third trimester, the preferred timing for fetal MRI is the 32nd week of gestation at that time one can make a comprehensive structural assessment [24]

Fetal MRI added additional information in cases of neck anomalies as in cases of cystic hygroma and cervical teratoma in which MRI was superior in assessing the nature of the lesion solid with cystic changes, the extension of the lesion, and the relation to oropharyngeal airway.

The nature of the lesion (cystic, vascular, neoplastic, etc.) and its extension can be detected accurately with fetal MRI. Accordingly, MRI can assess the severity of the malformation by assessing its actual extension, whether confined to the neck or had mediastinum invasion [25].

In the US, neck mass extension and upper airway scanning are challenging. On T2-W fetal MRI the airway appears typically as hyperintense fluid-filled passage so that T2WI can assess the degree of the obstruction, its invasion to the mediastinum, and also shows esophageal and/or vascular compression, which can negatively affect the prognosis [26]

Cleft lip/palate $(\mathrm{CL} / \mathrm{P})$ is the commonest congenital malformations affecting the face. Recently, there has been a wide acceptance to use the US in diagnosing $\mathrm{CL} / \mathrm{P}$. Jean-Marc et al. [27] described high rates of recognition (up to 85\%) of fetal facial anomalies using the US. In comparison, MRI offers stronger tissue contrast, multiplanar capability, and higher resolution. Also, MRI is less affected by human influences. Moreover, it has been found that MRI can aid in the diagnosis of fetal cleft lip and/or cleft palate. Additionally, based on MRI, CL/P classification, and other integrated anomalies can be determined, helping in perinatal follow-up and management. [28] 
The limitation of this study was the small number of fetuses studied due to limited knowledge about fetal MRI, as it would be more beneficial if it was performed on a large-scale multicenter study in addition to financial issues with MRI being an expensive technique in comparison to the US. Last but not least, the lack of postnatal autopsy in our country due to religious factors makes it impossible to compare MRI to open procedure confirming the proposed diagnosis.

As MRI is not conducted in negative US cases, there is deficient evidence in assessing the true negative and false negative cases on fetal MRI, leading to uncertainty of the sensitivity, specificity, and predictive values.

\section{Conclusion}

Fetal MRI raises confidence in non-CNS malformation assessment. Compared to the US, MRI overcomes many of the obstacles faced by the antenatal US. MRI is superior to the US in refining, changing, or adding more diagnostic information about the disease.

Also, MRI can guide management plans and influence parental counseling. In a large number of non-fatal nonCNS anomalies, fetal MRI can provide an accurate and timely diagnosis that may help in organizing a suitable therapeutic intervention, which can improve the survival and the quality of postnatal life. Hence, when the US suspected or diagnosed non-CNS anomalies, we recommend fetal MRI to be used in the diagnostic workup. The combined MRI and ultrasound provide the highest accuracy.

\begin{abstract}
Abbreviations
US: Ultrasound; CNS: Central nervous system; ROI: Region of interest; PUV: Posterior urethral valve; ARPKD: Autosomal recessive polycystic kidneys; CCAM: Congenital cystic adenomatoid malformation; MCDK: Multicystic dysplastic kidneys; PUJO: Pelvi-ureteric junction obstruction; $\mathrm{CDH}$ : Congenital diaphragmatic hernia; CL/P: Cleft lip/palate; SSFSE: Single-shot fast SE sequence; SSFP: Steady-state free precession; T1-WI:T1-weighted sequence; DWl: Diffusionweighted image; MKS: Meckel-Gruber syndrome; U-V: Ureterovesical.
\end{abstract}

\section{Acknowledgements}

Not applicable

\section{Authors' contributions}

SE designed the study, collected the data, interpreted the MR image, carried out the statistical analysis, and wrote the manuscript. MHZ, AME, and TMA edited the manuscript and revised the sequence alignment. HH interpreted the MR images, edited the manuscript, and revised the sequence alignment. All authors read and approved the final manuscript.

\section{Funding}

No sources of funding (not applicable).

\section{Availability of data and materials}

All data supporting the study are presented in the manuscript or available upon request.

\section{Declarations}

\section{Ethics approval and consent to participate}

This study was approved by the Research Ethics Committee of the Faculty of Medicine at Alexandria University in Egypt on 2018 (reference number is 0201132, IRB number 00012098). Written informed consent was obtained from all patients included in this study.

\section{Consent for publication}

Not applicable.

\section{Competing interests}

The authors declare that they have no competing interests.

\section{Author details}

${ }^{1}$ Department of Diagnostic and Interventional Radiology, Faculty of Medicine, Alexandria University Hospital, Champollion Street, El Azareeta, Egypt ${ }^{2}$ Department of Obstetrics and Gynecology, El Shatby Maternity University Hospital, Faculty of Medicine, Alexandria University Hospital, Port-Said Street, El-Shatby 21526, Egypt.

Received: 18 November 2021 Accepted: 22 January 2022

Published online: 10 February 2022

\section{References}

1. Rao BG, Ramamurthy B (2009) Pictorial essay: MRl of the fetal brain. Indian J Radiol Imaging 19(1):69

2. Coakley FV, Glenn OA, Qayyum A, Barkovich AJ, Goldstein R, Filly RA (2004) Fetal MRl: a developing technique for the developing patient. Am J Roentgenol 182(1):243-252

3. Malinger $G$, Lev D, Lerman-Sagie $T$ (2002) Is fetal magnetic resonance imaging superior to neurosonography for detection of brain anomalies? Ultrasound Obstet Gynecol 20(4):317-321

4. Twickler DM, Magee KP, Caire J, Zaretsky M, Fleckenstein JL, Ramus RM (2003) Second-opinion magnetic resonance imaging for suspected fetal central nervous system abnormalities. Am J Obstet Gynecol 188(2):492-496

5. Gupta S, Mohi JK, Gambhir P, Mohi MK (2020) Prenatal diagnosis of congenital anomalies of genito-urinary system on fetal magnetic resonance imaging. Egypt J Radiol Nucl Med 51(1):1

6. Gonçalves LF, Lee W, Mody S, Shetty A, Sangi-Haghpeykar H, Romero R (2016) Diagnostic accuracy of ultrasonography and magnetic resonance imaging for the detection of fetal anomalies: a blinded case-control study. Ultrasound Obstet Gynecol 48(2):185-192

7. Griffiths PD, Porteous M, Mason G, Russell S, Morris J, Fanou EM, Reeves MJ (2012) The use of in utero MRI to supplement ultrasound in the foetus at high risk of developmental brain or spine abnormality. Br J Radiol 85(1019):e1038-e1045

8. Hosny IA, Elghawabi HS (2010) Ultrafast MRI of the fetus: an increasingly important tool in prenatal diagnosis of congenital anomalies. Magn Reson Imaging 28(10):1431-1439

9. Furey EA, Bailey AA, Twickler DM (2016) Fetal MR imaging of gastrointestinal abnormalities. Radiographics 36(3):904-917

10. Bakare VN, Taori K, Disawal A, Rathod J, Dhakate S, Wavare PS (2013) Role of MRI in suspected cases of congenital fetal anomalies on ultrasound. In: European congress of radiology-ECR

11. Marine MB, Forbes-Amrhein MM (2020) Magnetic resonance imaging of the fetal gastrointestinal system. Pediatr Radiol 50(13):1895-1906

12. Chen CP, Cheng SJ, Sheu JC, Lin YH (2004) Third-trimester evaluation of choledochal cyst using magnetic resonance imaging. Prenat Diagn 24(10):838-839

13. Chalouhi GE, Millischer AÉ, Mahallati H, Siauve N, Melbourne A, Grevent D, Vinit N, Heidet L, Aigrain Y, Ville Y, Blanc T (2020) The use of fetal MRI for renal and urogenital tract anomalies. Prenat Diagn 40(1):100-109

14. Brewerton LJ, Chari RS, Liang Y, Bhargava R (2005) Fetal lung-to-liver signal intensity ratio at MR imaging: development of a normal scale and possible role in predicting pulmonary hypoplasia in utero. Radiology 235(3):1005-1010 
15. Kumari N, Pradhan M, Shankar VH, Krishnani N, Phadke SR (2008) Postmortem examination of prenatally diagnosed fatal renal malformation. $J$ Perinatol 28(11):736-742

16. Alamo L, Laswad T, Schnyder P, Meuli R, Vial Y, Osterheld MC, Gudinchet F (2010) Fetal MRI as complement to US in the diagnosis and characterization of anomalies of the genito-urinary tract. Eur J Radiol 76(2):258-264

17. Said AH, El-Kattan E, Abdel-Hakeem AK, Saleem S (2016) In utero MRI diagnosis of fetal malformations in oligohydramnios pregnancies. Egypt J Radiol Nucl Med 47(4):1733-1742

18. Malguria NN, Merchant SA, Kiran KV, Verghese SL (1996) Meckel-Gruber syndrome associated with short limbed dwarfism. J Postgrad Med 42(2):55

19. Barseghyan K, Jackson HA, Chmait R, De Filippo RE, Miller DA (2008) Complementary roles of sonography and magnetic resonance imaging in the assessment of fetal urinary tract anomalies. J Ultrasound Med 27(11):1563-1569

20. Raga F, Osborne NG, Bonilla F, Bonilla-Musoles F, Machado LE, Ruiz F, Castillo JC (2010) Prune-belly syndrome versus posterior urethral valve. Donald Sch J Ultrasound Obstet Gynecol 4(4):405-417

21. Manganaro L, Perrone A, Sassi S, Fierro F, Savelli S, Di Maurizio M, Tomei A, Francioso A, La Barbera L, Giancotti A, Ballesio L (2008) Diffusionweighted MR imaging and apparent diffusion coefficient of the normal fetal lung: preliminary experience. Prenat Diagn 28(8):745-748

22. Bekiesińska-Figatowska M, Romaniuk-Doroszewska A, Brągoszewska H, Iwanowska B, Szkudlińska-Pawlak S, Mądzik J, Duczkowski M, Krupa K, Cabaj A, Kwaśniewicz P, Duczkowska A (2018) Seventeen years of prenatal magnetic resonance imaging at the Institute of Mother and Child in Warsaw. Polish J Radiol 83:e94

23. Berrocal T, Madrid C, Novo S, Gutiérrez J, Arjonilla A, Gómez-León N (2004) Congenital anomalies of the tracheobronchial tree, lung, and mediastinum: embryology, radiology, and pathology. Radiographics 24(1):e17

24. Zemet R, Amdur-Zilberfarb I, Shapira M, Ziv-Baran T, Hoffmann C, Kassif E, Katorza E (2020) Prenatal diagnosis of congenital head, face, and neck malformations-is complementary fetal MRl of value? Prenat Diagn 40(1):142-150

25. Tonni G, Granese R, Santana EF, Parise Filho JP, Bottura I, Peixoto AB, Giacobbe A, Azzerboni A, Júnior EA (2017) Prenatally diagnosed fetal tumors of the head and neck: a systematic review with antenatal and postnatal outcomes over the past 20 years. J Perinat Med 45(2):149-165

26. Taghavi K, Berkowitz RG, Fink AM, Farhadieh RD, Penington AJ (2012) Perinatal airway management of neonatal cervical teratomas. Int J Pediatr Otorhinolaryngol 76(7):1057-1060

27. Levaillant JM, Nicot R, Benouaiche L, Couly G, Rotten D (2016) Prenatal diagnosis of cleft lip/palate: the surface rendered oro-palatal (SROP) view of the fetal lips and palate, a tool to improve information-sharing within the orofacial team and with the parents. J Cranio-Maxillofac Surg 44(7):835-842

28. Zheng W, Li B, Zou Y, Lou F (2019) The prenatal diagnosis and classification of cleft palate: the role and value of magnetic resonance imaging. Eur Radiol 29(10):5600-5606

\section{Publisher's Note}

Springer Nature remains neutral with regard to jurisdictional claims in published maps and institutional affiliations.

\section{Submit your manuscript to a SpringerOpen ${ }^{\circ}$ journal and benefit from:}

- Convenient online submission

- Rigorous peer review

- Open access: articles freely available online

- High visibility within the field

- Retaining the copyright to your article

Submit your next manuscript at $\boldsymbol{\nabla}$ springeropen.com 